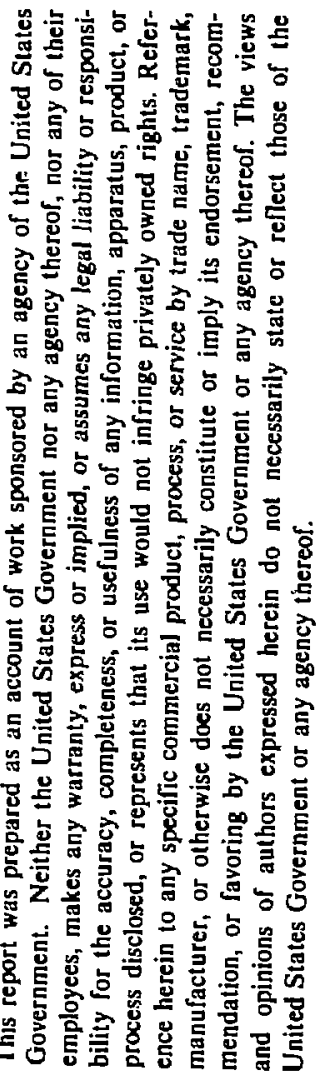

\section{Programmable CCD Imaging System for Synchrotron Radiation Studies(*)}

Brian Rodricks and Christine Brizard Advanced Photon Source Argonne National Laboratory Argonne, IL 60439
ANL/CP- -73528

DE91 017303 tris.

AUO 211991

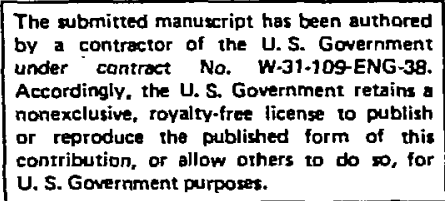

*This work supported by the U.S. Department of Energy, BES-Materials Sciences, under contract no. W-31-109-ENG-38

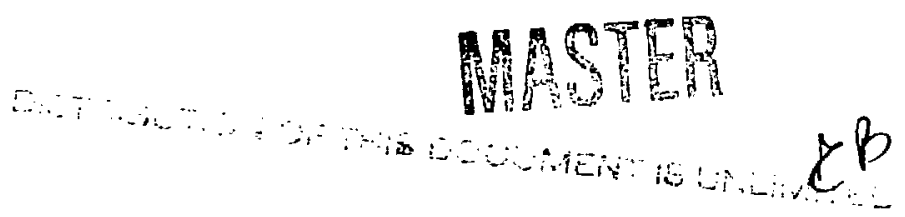




\title{
Programmable CCD Imaging System For Synchrotron Radiation Studies
}

\author{
Brian Rodricks and Christine Brizard \\ Advanced Photon Source \\ Argonne National Laboratory \\ 9700 S. Cass Avenue \\ Argonne, IL 60439
}

\begin{abstract}
A real-time imaging system for $\mathrm{x}$-ray detection has been developed. The CAMAC-based system has a Charge Coupled Device (CCD) as its active detection element. The electronics consist of a CAMAC-crate-based dedicated microprocessor coupled to arbitrary waveform generators, programmable timing, and ADC modules. The hardware flexibility achievable through this system enables one to use virtually any commercially available $\mathrm{CCD}$. A dedicated CAMAC-based display driver allows for real-time imaging on a high-resolution color monitor. An optional front end consisting of a fiber-optic taper and a focusing optical lens system coupled to a phosphor screen allows for large area imaging. Further, programming flexibility, in which the detector can be used in different read-out modes, enables it to be exploited for time-resolved experiments. In one mode, sections of the CCD can be read-out with millisecond time-resolution and, in another, the use of the CCD as a storage device is exploited resulting in microsecond time-resolution. Three different CCDs with radicaily different read-out timings and waveforms have been tested: the TI 4849 , a 390x584 pixel array; TC 215 , a 1024x1024 pixel array; and the TH 7883, a 576x384 pixel array. The TC 215 and TI 4849 are single-phase CCDs manufactured by Texas Instruments, and the TH 7883 is a four-phase device manufactured by Thomson-CSF. The CCD characterized for uniformity, charge transfer efficiency (CTE), linearity, and sensitivity is the TC215.
\end{abstract}




\section{INTRODUCTION}

Three third generation synchrotron radiation sources are currently under construction: Advanced Photon Source (APS) in U.S.A., European Synchrotron Radiation Facilities (ESRF) in France, and Synchrotron Positron Ring (SPRING) in Japan. The use of insertion devices (wigglers, undulators) will make APS, in 1995, an x-ray source 10,000 times brighter than any $x$-ray source existing today.[1] The high brilliance of these new sources opens new and exciting fields, especially in time-resolved $x$-ray experiments. [2] The success of innovative scientific techniques relies on the use of appropriate detectors. Requirements that will allow a detector to satisfy a broad spectrum of science are twodimensionality, high dynamic range, fast response, linearity, sensitivity, low noise, radiation hardness, and versatility. Presently Charge Coupled Devices (CCDs) have characteristics that match these severe requirements very well.[3] Instrumentation projects at APS currently include the development of a programmable CCD detector that can adapt to the changing market of CCD chips. This calls for the development of an extremely flexible and versatile system. The system developed uses a Computer Automated Measurement and Control (CAMAC)-based electronics and a 64-MegaByte hard memory MicroVax III computer. The architecture of this system enables the use of CCD chips from various manufacturers, and the large memory helps-in=time-resolved experimentation-So far, three different chips have been successfully tested and used with visible light or direct x-ray illumination: Texas Instruments TI 4849, TI TC 215, and Thomson TH 7883 (Table I). The detector has already been used at various synchrotron radiation facilities to perform imaging and time-resolved experiments from minute to microsecond time-scale. 


\section{CHARGE COUPLED DEVICES (CCDs)}

The CCD is basically an integrated-circuit device that uses an array of photosensitive elements to convert incident illumiration into a proportional electrical charge. Each pixel stores its charge in a metal-oxide semiconductor (MOS) capacitor, a potential well. A parallel clocking operation shifts each row of charge down the array with the first row of data entering a serial register. From the serial register, a serial clock performs a pixel by pixel sequential transfer to an on-chip amplifier. ${ }^{[4]}$ The operation of a CCD is composed of a fast clear sequence where all pixels are cleared by a series of parallel transfers. This is followed by an integration period during which charges proportional to the amount of incident photons are created and stored in each pixel. Then, the read-out is performed by a succession of parallel transfers that are interlaced with a sequence of serial transfers. Finally, the reset operation clears the on-chip amplifier after each serial transfer.

Two of the chips used in this programmable imaging detector are virtual phase devices. An asymmetric well structure in this type of CCD is achieved by deep ion implants so that only a single level of external clocking electrodes is required.[5] The TI 4849 has 390 columns and 584 rows of $22.4 \mathrm{~mm}^{2}$ pixel and needs 3 waveforms to be read-out: parallel, serial, and reset clocks. The TI TC 215 has $1024 \times 102412 \mathrm{~mm}^{2}$ pixels and needs 8 waveforms to be read-out: 2 parallel, 2 serial, 2 reset, 1 transfer gate, and 1 antiblooming gate clocks. This chip has 2 serial registers that enable it to read the CCD twice as fast. The other chip used is the TH 7883 (Thomson-France), which has $576 \times 38423 \mathrm{~mm}^{2}$ pixels and needs 7 waveforms to be read-out: 4 parallel, 2 serial, and 1 reset clocks.[6] In addition to the number of waveforms being different, the timings, amplitudes, and shapes are totally different. The logic of the electronics has been designed to satisfy the requirements with diversity of this magnitude. 


\section{PROGRAMMABLE DETECTOR}

Fig. 1 is a block diagram of the CAMAC-based electronic system. The CAMAC crate houses the arbitrary waveform generators, a $20 \mathrm{MHz}$ master clock, a 12-bit ADC module, a 12bitx1M memory module, a display driver, and a LSI-11 dedicated microprocessor. Global control is accomplished by a MicroVax III computer. Communication between the computer and the LSI is through encoded interrupt bit patterns in the form of Look-At-Me's (LAMs) received by the interrupt register. The key to the flexibility relies on the interaction between the MicroVax III computer, the LSI-11 microprocessor, and the master clock. For each required waveform, timing and voltage are downloaded into the waveform generator memory banks. These waveform generator modules are triggered by the master programmable clock, which is, in turn, commanded by the LSI-11 microprocessor. The waveform generators know the number of columns, i.e., the number of serial transfers to perform, and the LSI-11 knows the number of rows, i.e., the number of parallel transfers to perform. A set of flags between the microprocessor and the master clock assures the complete reading of the chip. After completion of a frame read-out, the LSI-11 informs the MicroVax and waits for the next task to perform.

The CCD output data are preamplified, digitized by a 12-bit ADC, and stored in a 1 Mbyte buffer memory. This data can be directly displayed on a high-resolution video monitor (1024x1024) through a high-resolution display driver:-The-24=bit high-resolution=display

driver can perform color contour mapping of 256 simultaneous colors from a pallette of 16 million. It can also display intensity profiles of row and column data. This feature is very convenient for on-line diagnostics during experiments. The data, when necessary, are stored on the 778 Mbyte computer hard disk. 
This flexible system allows use of a CCD chip in 3 different modes. In the first mode, a fuli frame can be read and stored in buffer memory in one second. The integration time can be as short as a few microseconds or as long as a few minutes in a latch mode. The other two modes, dedicated for direct $\mathrm{x}$-ray exposures, exploit the serial/parallel read-out of the $\mathrm{CCD}$. For the second mode, slits a few rows wide are physically put in front of the chip. The charges stored in the few exposed rows are transferred down by successive parallel transfers to the serial register where they are read-out. This method is used to perform fast experiments in the millisecond range. Further, since the parallel transfer is much faster than the serial transfer, rows of data can be added in the serial register to improve statistics without any significant increase in time. The 20 -millisecond time resolution limit is due to the data transfer time from the buffer memory to the computer hard disk. For the third mode, the $C C D$ is used as a storage device where slits, if possible one row wide, are placed in front of the top of the chip. Parallel transfers bring down the charges until they reach the serial register. Then, the whole frame is read. This mode is used for ultra-fast experiment in the microsecond range. The 2 -microsecond time resolution limit is due to the high CCD row capacitance value. Software written for each chip allows one to change chips and modes of transfer with no down time.

The CCD chip is placed on a customized PC board connecting the chip pins with the signal wires from the CAMAC crate. The chip, surrounded by the board, is put on a three-stage Peltier cooler (which provides a - $40 \mathrm{deg}$. $\mathrm{C}$ temperature) and is housed in a vacuum chamber to prevent condensation (Fig. 2). For direct x-ray imaging, a beryllium window vacuum chamber is used. For visible light imaging, a quartz window vacuum chamber is used coupled to an optical system and a phosphor screen. In the visible light spectrum, the quantum efficiency of CCD chips increases with the wavelength.[7] So, for imaging purposes, phosphors with green light emission are more appropriate than those with blue light emission. The phosphor $\mathrm{Gd}_{2} \mathrm{O}_{2} \mathrm{~S}: \mathrm{Tb}$ (Trimax-3M) has, for example, been used for 
spectroscopy experiments. [8] For time-resolved experiments, the decay time of the phosphor must be smaller, of course, than the experiment time constant. The scintillator decay time increases with the emission wavelength. All time-resolved experiments were done with direct $x$-ray exposures. For imaging experiments, the optical magnification or demagnification system includes a combination of lenses, fiber-optics taper, and bellows. [9] The choice of these different components depends on the required magnification or demagnification ratio (Fig. 3). The lenses are Nikon $50 \mathrm{~mm} / \mathrm{F} 1.2$ and 200 $\mathrm{mm} / \mathrm{F} 4$. The SCHOTT fiber-optics taper has a 52-mm diameter on the larger side and a 13$\mathrm{mm}$ diameter on the smaller one: hence, the magnification/ demagnification ratio is 4 . The length of the fiber-optics taper is $55 \mathrm{~mm}$. The use of stray light absorbing elements (Extra Mural Absorption) and adequate optical insulation (cladding) between the fibers provide optimum image contrast. The smaller end of a fiber optic is approximately $2 \mu \mathrm{m}$. The optical system magnification ratio can be as high as 16 . In this case, the intrinsic resolution of the $\mathrm{CCD}$ camera is less than one micron.

In order for the device to function as an $\mathrm{x}$-ray detector for scientific purposes, it must have very high charge transfer efficiency, linearity, uniformity of response, quantum efficiency $(\mathrm{QE})$, and dynamic range. The electronic system described above allows one to fine tune the read-out waveforms for maximum charge transfer efficiency (CTE). The measurements were done by exposing the detector to a uniform source of light and using the "extendedpixel-edge-response": (EPER), $[10]$ in which

$$
C T E=\left[1-q_{d} /\left(q_{i} N_{p}\right)\right],
$$

where $q_{d}$ is the net deferred charge in the extended region, $q_{i}$ is the signal level of the last column, and $\mathrm{N}_{\mathrm{p}}$ is the number of pixels transferred. 
Fig. 4 is a plot of the measured CTE across each row. The data indicate that the CTE is better than 0.99999 . Another advantage of a programmable waveform system is that the CTE degradation due to radiation damage can be compensated, to some extent, by waveform adjustment.

Linearity measurements were performed using a light emiting diode (LED) as the source.

This light was focused onto the $\mathrm{CCD}$, and the intensity as a function of time was recorded as shown in Fig. 5. Data indicate that the system response is linear to within $0.5 \%$ over a typical operating range where the values correspond to midway for the ADC saturation (about 2000 adc units).

The quantum efficiency depends on the thickness of the depletion depth and the energy of the $\mathrm{x}$-ray photon. The thickness of Si required for $90 \%$ quantum efficiency (QE) for 0.5 $\mathrm{keV}$ to $5 \mathrm{keV}$ ranges from $0.5 \mu \mathrm{m}$ to $50 \mu \mathrm{m}$.[11] Typical depletion depths of CCDs range from 1 to $15 \mu \mathrm{m}$, resulting in low $Q E$ for high energy $x$-rays. Further, for low energy $x$ rays, the $\mathrm{QE}$ is limited by the dead layer of oxide and the gate electrodes present on the front surface of the CCD. The depletion depth of the TI 4849 is $12 \mu \mathrm{m}$, which makes the device approximately $10 \% \mathrm{QE}$ for direct $\mathrm{x}$-ray absorption at $7 \mathrm{keV}$.

\section{RADIATION DAMAGE IN CCDS}

A critical consideration when choosing CCDs to be used with direct $\mathrm{x}$-rays at a synchrotron source facility is the possibility of radiation damage. General CCD radiation damage includes bulk and ionization effects. [10] Synchrotron radiation doesn't usually create bulk effects that would require a $150 \mathrm{keV}$ electron to displace a silicon atom in the bulk lattice. The presence of this effect would have a profound impact on the CTE performance. 
However, ionization effects must be considered. Ionization-induced damage results in a buildup of charge in the CCD's gate insulaior. Only a few eV of energy are needed to alter the flat-band characteristics of the gate insulator. Moreover, the presence of interface states between the gate oxide and bulk silicon leads to an increase in dark current generation. The TI 4849 chip has a depletion depth of approximately $12 \mu \mathrm{m}$, which is very appropriate for $\mathrm{x}$-ray energy in the range of 4-8 keV. This chip was radiation-tested on a bending magnet beamline (X16 at the National Synchrotron Light Source-NSLS) at an energy of $8 \mathrm{keV}$ for about 20 hours of continuous use. The estimated absorbed dose is $100 \mathrm{krad}$. As expected, the CTE, estimated with the "extended-pixel-edge-response" (EPER) method, was not measurably degraded in the exposed region of the chip compared to that in the mask area. The good performance of this virtual-phase CCD chip in the presence of synchrotron radiation is more evident by the absence of significant increase in the dark current. In case of a higher radiation dose, which would create a dark current increase, the flexibility of this programmable detector would allow one to change the waveform voltages in order to minimize the dark signal.

\section{CONCLUSION}

-A programmable 2-dimensional- $E$ CD=detector for-synchirntron instrumentation has been developed for visible light or direct $\mathrm{x}$-ray imaging. The tremendous flexibility of this camera allows the use of any kind of CCD chips, and so in different time-resolution modes. Even larger CCD chips $(2048 \times 2048$ or $4096 \times 4096)$ could be used or a quad-CCD chip combination for large imaging purposes, such as in biology or medicine. Timeresolved $\mathrm{x}$-ray experiments can be performed with a time resolution up to two microseconds. The combination of fast parallel detectors and the new generation of 
synchrotron radiation sources opens new techniques in $x$-ray diffraction, microradiography, microtomography, and topography.

\section{ACKNOWLEDGEMENTS:}

It is a pleasure to thank Gopal Shenoy and Dennis Mills for their great support; also Joe Arko and Ron Hopf for their invaluable help assisting us in building the detector. Roy Clarke, Walter Lowe, and Bob McHarrie have been actively involved in the time-resolved experiments performed at NSLS. This work is supported by U.S. Dept. of Energy, BESMaterials Science, under grant contract \#W-31-109-ENG-38.

${ }^{1}$ G.K. Shenoy, P.J. Viccaro, D.M. Mills, Characteristics of the 7-GeV Advanced Photon Source: A Guide for Users.

2D.M. Mills, Time-Resolved X-Ray Studies Using Synchrotron Radiation, Handbook on Synchrotron Radiation, Volume 3,ed. D. Moncton, Elsevier Science, 1991.

${ }^{3}$ Sol M. Gruner, Rev. Sci. Instrum. 60(7), 1545-1551, (July 1989).

${ }^{4}$ B. Rodricks, R. Clarke, R. Smither, A. Fontaine, Rev. Sci. Instrum. 60 (8), 2586-2591, (August 1989).

5 J. Hynecek, IEEE Trans. Electron Dev. 28, 483 (1981).

6B. Rodricks, C. Brizard, Programmable CCD Imaging System For Synchrotron Radiation Studies, submitted to NIM.

7J. Janesick, M. Blouke, Sky \&Telescope, 238-242, (Sept. 1987). 
8 J.C. Campuzano, G. Jennings, L. Beaulaigue, B. Rodricks, C. Brizard, Soft X-ray Spectro-microscopy, Proc. SPIE's 1990 Int. Symposium on Optical and Optoelectronic Applied Science and Engineering, San Diego, 1990.

${ }^{9}$ C. Brizard, B. Rodricks, Programmable CCD Imaging System, submitted to Optical Engineering.

10J. Janesick, T. Elliott, F. Pool, Radiation Damage in Scientific Charge-Coupled Devices, IEEE, 1988 Nuclear Science Symposium, Orlando, FL,11/9-11/88.

${ }^{11}$ R. Germer, SPIE 491, 434 (1984). 
Table I. Performance characteristics of the three CCDs

Device

TI 4849

TC 215

TH 7883

$\begin{array}{llll}\text { Phase } & \text { Virtual } & \text { Virtual } & \text { Four } \\ \text { Frame Size } & 390 \times 584 & 1024 \times 1024 & 576 \times 384 \\ \text { Readout Waveforms } & 3 & 8 & 7 \\ \text { Readout Noise } & 20 \text { e-/pixel } & 60 \text { e-/pixel } & 117 \text { e-/pixel } \\ \text { Sensitivity* } & 1 \text { ADU } & 1 \text { ADU } & 1 \text { ADU } \\ \text { Saturation } & 200,000 \text { e- } & 60,000 \mathrm{e}- & 1,000,000 \text { e- } \\ \text { Linearity } & <0.5 \% & <0.5 \% & <0.5 \% \\ \text { CTE } & >0.99996 & >0.99999 & >0.9999 \\ \text { Pixel Size }\left(\mu \mathrm{m}^{2}\right) & 22.4 & 12 & 23 \\ \text { Dynamic } R \text { Range } & 4000 & 4000 & 8000\end{array}$

* 1 ADU (analog to digital unit) above background 


\section{Figure captions:}

Fig. 1: Architecture of the programmable imaging system.

Fig. 2: Schematic of the CCD detector.

Fig. 3: CCD detector: A) vertical height mounting assembly, B) water-cooled base, C) heat sink, D) Peltier cooler, E) vacuum chamber, F) CCD chip

Fig. 3a: Demagnifying geometry: G) $50 \mathrm{~mm} / \mathrm{F} 1.2$ Lens, H) $200 \mathrm{~mm} / \mathrm{F}$ 1.4 Lens, J) Fiber optics taper, K).Phosphor.

Fig. 3b: Magnifying geometry: I) Bellows.

Fig. 4: Charge transfer efficiency vs. row.

Fig. 5: Linearity. 


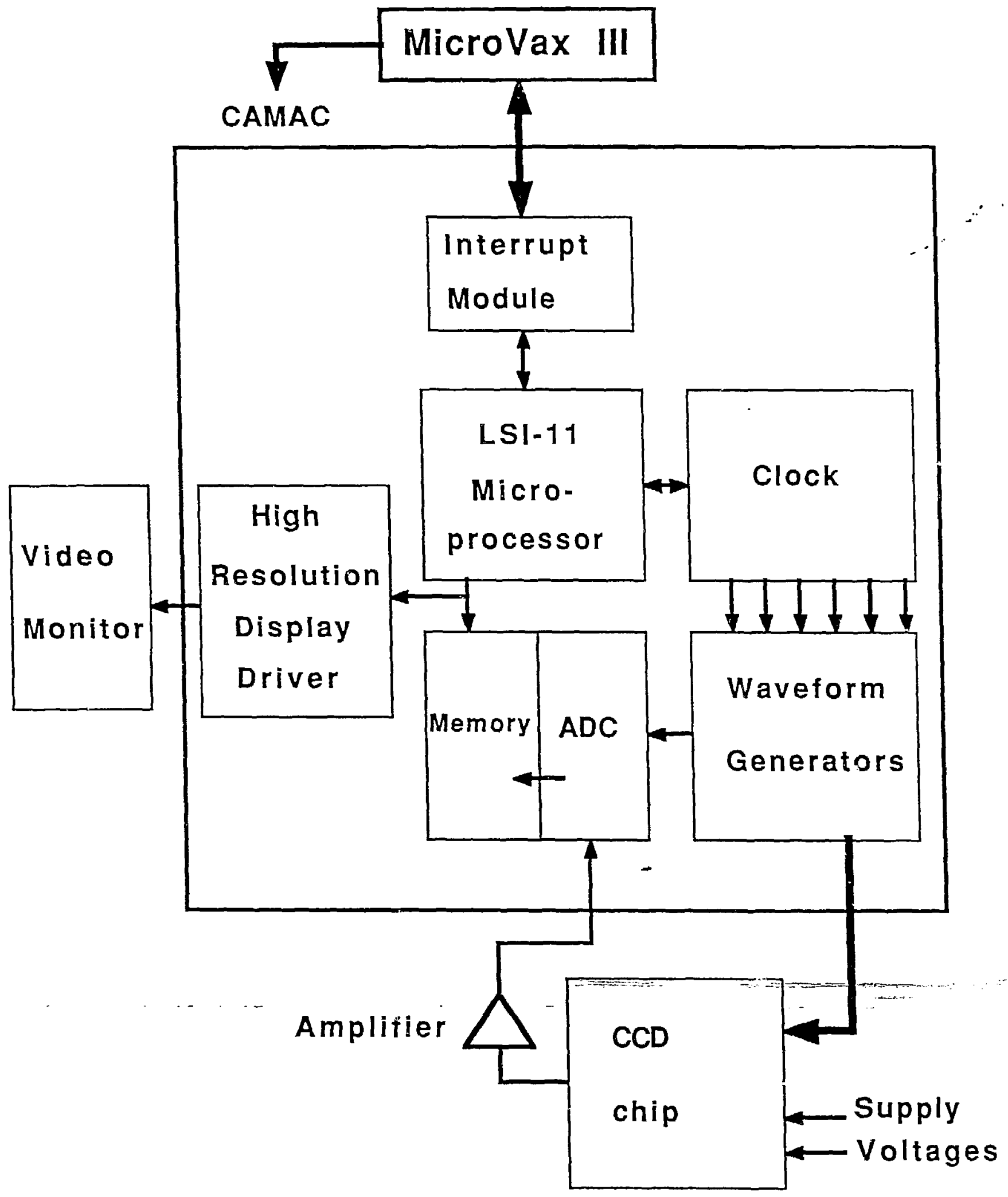




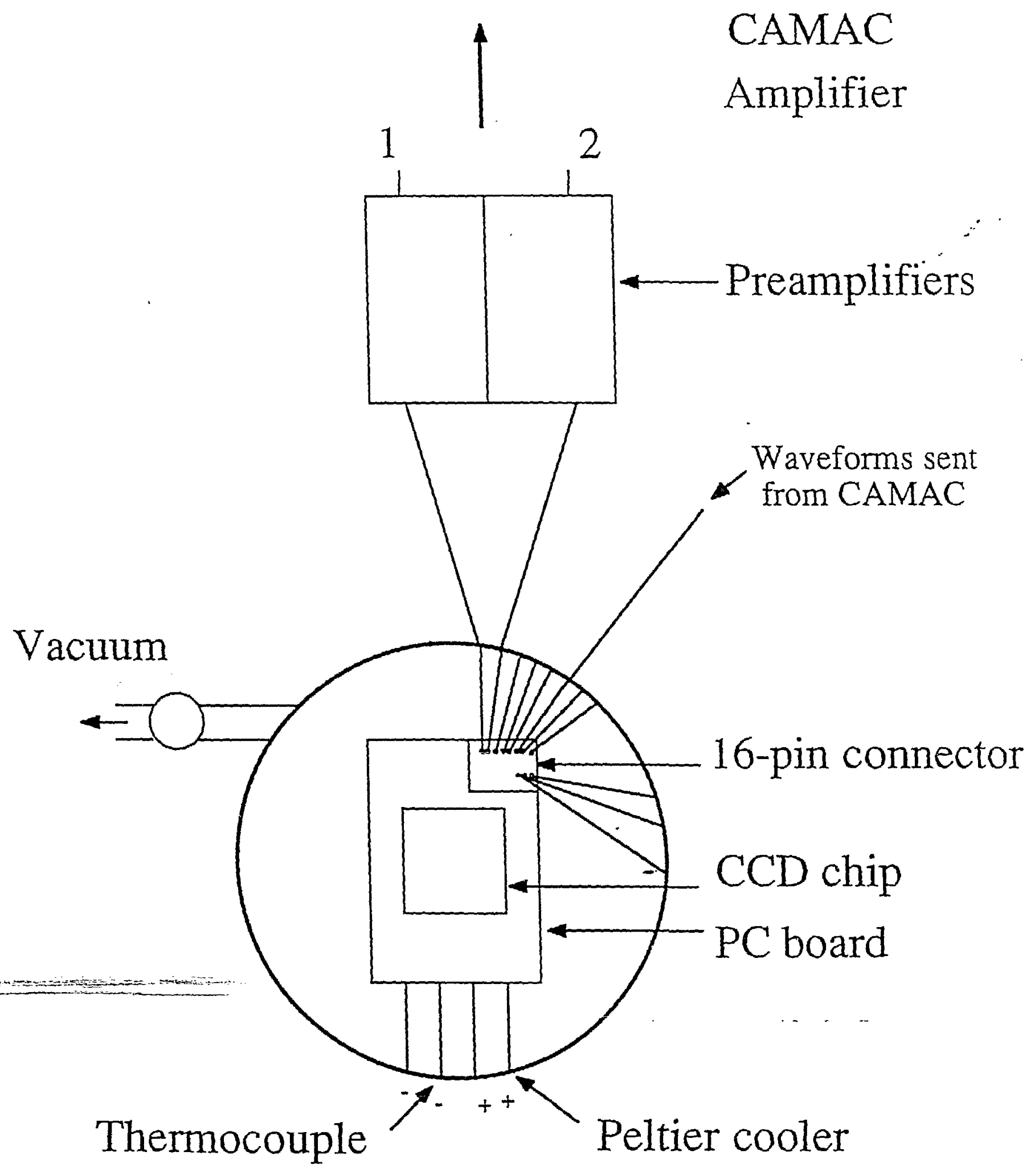




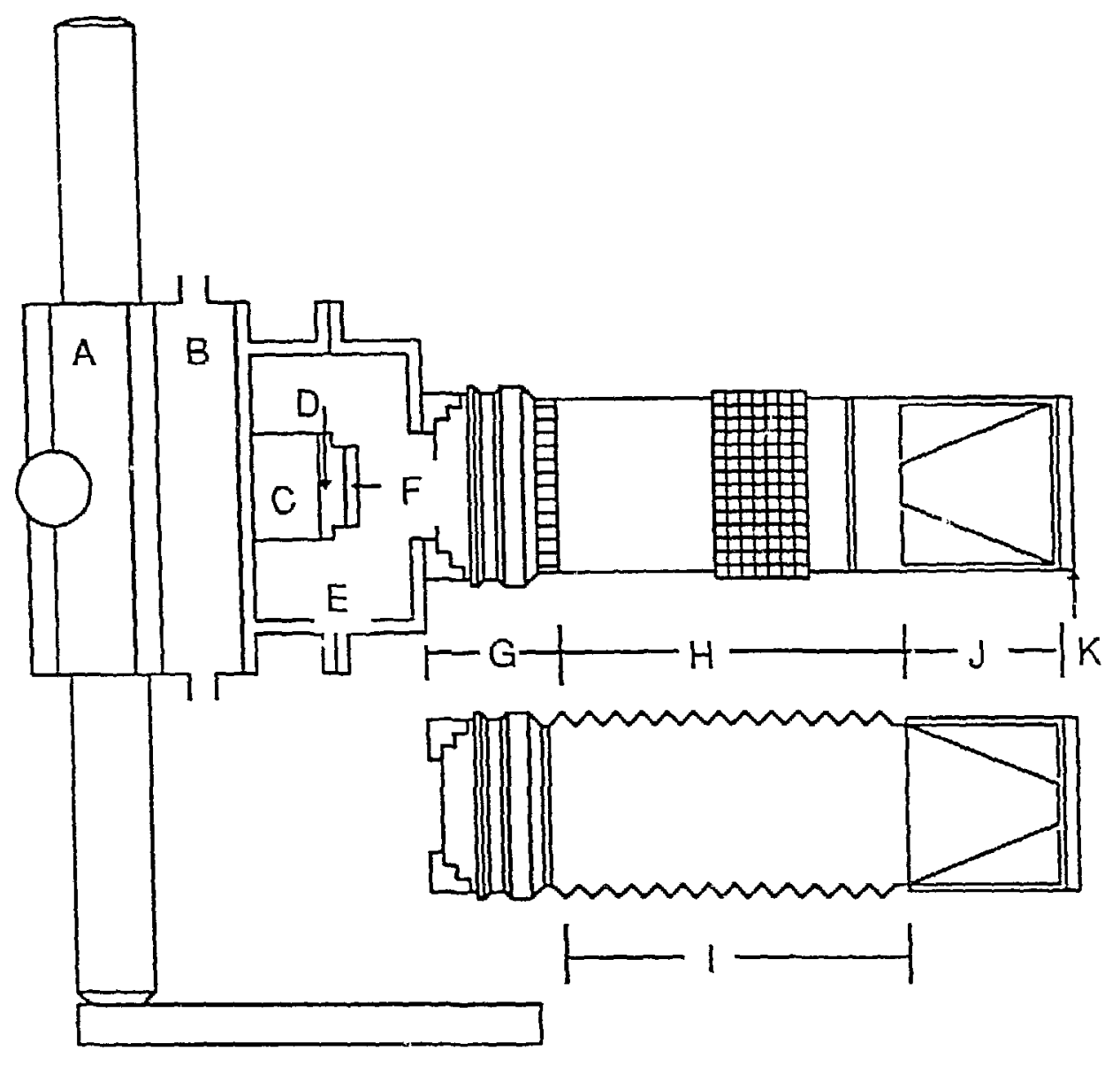




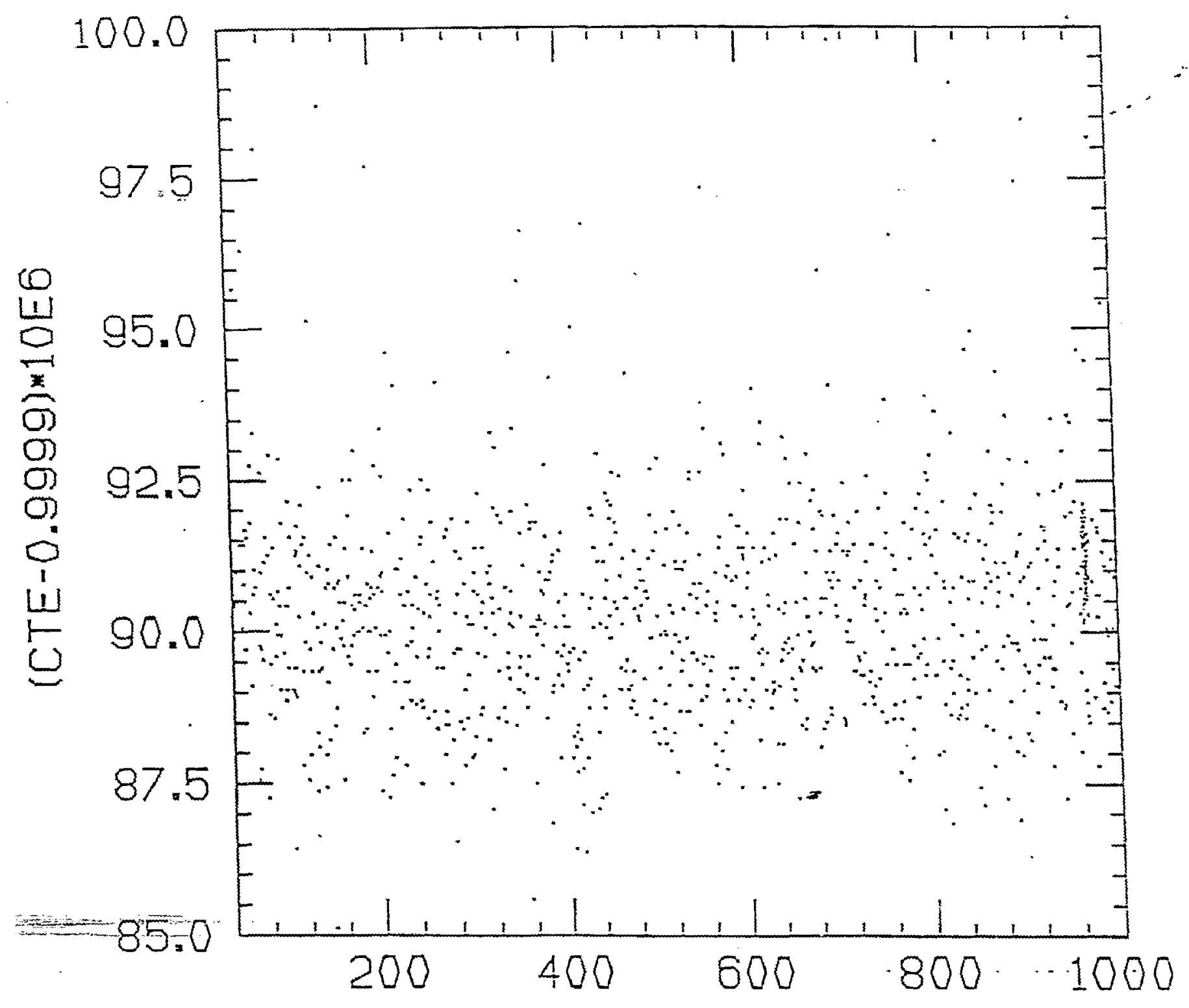

Row number 


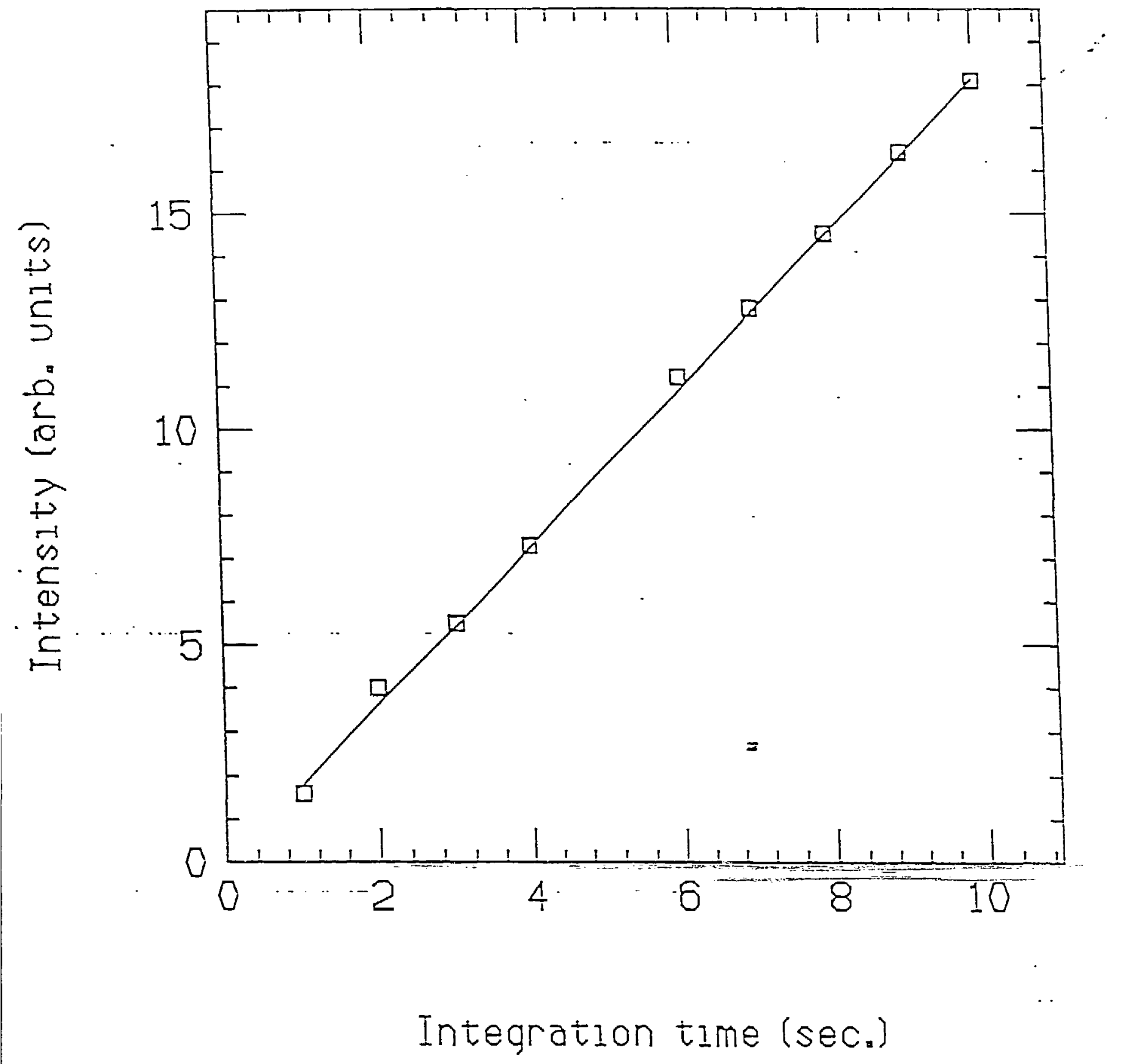

